\title{
Dimension estimation-based spectrum sensing for cognitive radio
}

\author{
Bassem Zayen $^{1 *}$ and Aawatif Hayar ${ }^{2}$
}

\begin{abstract}
In this article, we will derive closed-form expressions of false alarm probabilities for a given threshold for the dimension estimation-based detector (DED) using Akaike information criterion (AIC) and the minimum description length (MDL) criterion. Specifically, the DED algorithm will be formulated as a binary hypothesis test using AIC and MDL curves. Based on the proposed statistic test, we will express the probability of false alarm of the DED algorithm for a fixed threshold using the cumulative density function for the distribution of Tracy-Widom of order two. The derived analytical decision thresholds are verified with Monte-Carlo simulations and a comparison between simulation and analytical results to confirm the theoretical results are presented. These results confirm the very good match between simulation and theoretic results.
\end{abstract}

Keywords: spectrum sensing, dimension estimation, threshold calculation

\section{Introduction}

The discrepancy between current-day spectrum allocation and spectrum use suggests that radio spectrum shortage could be overcome by allowing a more flexible usage of the spectrum. Flexibility would mean that radios could find and adapt to any immediate local spectrum availability. A new class of radios that is able to reliably sense the spectral environment over a wide bandwidth detects the presence/absence of legacy users (primary users) and uses the spectrum only if the communication does not interfere with primary users (PUs). It is defined by the term cognitive radio [1-3]. Cognitive radio (CR) technology has attracted worldwide interest and is believed to be a promising candidate for future wireless communications in heterogeneous wideband environments.

CR has been proposed as the means to promote efficient utilization of the spectrum by exploiting the existence of spectrum holes. The spectrum use is concentrated on certain portions of the spectrum while a significant amount of the spectrum remains unused. It is thus key for the development of CR to invent fast and highly robust ways of determining whether a frequency band is available or occupied. This is the area of

\footnotetext{
* Correspondence: bassem.zayen@eurecom.fr

${ }^{1}$ Mobile Communications Department, EURECOM, Sophia Antipolis, France Full list of author information is available at the end of the article
}

spectrum sensing for $\mathrm{CR}$ which will be considered in this article.

There are several spectrum sensing strategies that were proposed for CR. These strategies are categorized in two families: feature detection strategies and blind detection strategies. The feature detection approaches assume that a PU is transmitting information to a primary receiver when a secondary user (SU) is sensing the primary channel band. The elaboration of sensing techniques that use some prior information about the transmitted signal is interesting in terms of performance. In fact, feature detection algorithms employ knowledge of structural and statistical properties of PU signals when making the decision. The most known feature sensing technique is the cyclostationarity based detector (CD) [4]. Completely blind spectrum sensing techniques that do not consider any prior knowledge about the PU transmitted signal are more convenient to CR. A few methods that belong to this category have been proposed, but most of them suffer from the noise uncertainty and fading channels variations [5-8]. One of the most popular blind detectors is the energy detector (ED) [9]. This detector is the most common method for spectrum sensing because of its non-coherency and low complexity. [10] is an excellent reference on spectrum sensing methods. It gives a literature survey on feature detection and blind detection strategies. In this article, 
the $\mathrm{CD}$ and $\mathrm{ED}$ will serve as references when evaluating the performance of the dimension estimation-based detectors.

It is stated that current spectrum sensing techniques suffer from challenges in the low signal to noise range. The reasons for this have to be analyzed. It is suggested that information theoretic criteria is a possible area to look for a solution to overcome the problem. It is apparent that the problem at hand is wide and challenging. The initial attempt to apply information theoretic criteria for spectrum sensing was presented in $[11,12]$. The study presented in [11] suggested to use model selection tools like Akaike information criterion (AIC) and the minimum description length (MDL) criterion to conclude on the nature of the sensed band. These tools were used as detection rules for the dimension estimation detector (DED) [12]. AIC criterion was first introduced by Akaike [13] and Schwartz [14] for model selection. It was shown in [13] that the classical maximum likelihood principle can be considered to be a method of asymptotic realization of an optimum estimate with respect to a very general information theoretic criterion [13]. In [11,12], however, the AIC and MDL criterions were investigated in order to sense the signal presence. Specifically, the number of significant eigenvalues determined by the value which minimizes the AIC and/or MDL criterion was used as detection rule to decide on the presence/absence of data in the signal. The same idea was applied in $[15,16]$, published after [11], to develop two spectrum sensing algorithms exploiting the maximum or/and the minimum eigenvalue as detection rule. One is based on the ratio of the maximum eigenvalue to the minimum eigenvalue, the other is based on the ratio of the average eigenvalue to the minimum eigenvalue. However, in $[15,16]$, the model selection has not been considered. In [17], published in 2010, the authors used information theoretic criteria to detect PU presence. The proposed algorithm in [17] does not involve any threshold value when computing the false alarm probability. Otherwise, the false alarm probability derived in this work takes into account the threshold value. We will detail later, in Subsection 3.3, the difference between the proposed algorithm in this paper and the one derived in [17].

The study presented in $[11,12]$ were a preliminary step for this idea. Indeed, no threshold expression was given and the decision was taken using the values minimizing the AIC and/or MDL criterion computed by simulation. Also, in [12] all AIC and MDL values are computed to find the minimum values and to decide then on the availability of the PU band. In this article, however, we will simply compute the first and the second values of AIC and MDL to make this decision. For this purpose, we will present the DED detector as a binary hypothesis test. We will then give the exact threshold expressions of the DED detector using the two selection tools AIC and MDL. Specifically, we will derive closed-form expressions of false alarm probabilities for a given threshold using both AIC and MDL criterion. We will use in this derivation the cumulative density function (CDF) for the distribution of Tracy-Widom of order two [18]. The analytical results will be compared with simulation results.

The rest of this article is organized as follows. In Section 2, we will formulate the two users selection tools used throughout the development of the proposed algorithm. The DED algorithm will be presented in Section 3 using AIC and MDL criterion. We will derive in Section 4 closed-form expressions of false alarm probabilities for a given thresholds using both AIC and MDL criterion. Performance evaluation and advantages will be described in Section 5 and a comparison of the proposed detector with reference detectors will be given. The performance will be assessed under different conditions, using three simulation scenarios. Finally, Section 6 presents the conclusions of this article.

\section{Background of information theoretic criteria}

In this section, we will provide the background of information theoretic criteria. The general problem for model selection using information theoretic criteria is: given a set of $N$ observations $\left\{\mathbf{x}_{1}, \mathbf{x}_{2}, \ldots, \mathbf{x}_{N}\right\}$ and a family of operating models which are represented by a parameterized family of probability density functions $f$, determine the best fit model. The operating models are usually unknown, since only a finite number of observations is available. Therefore, approximating probability model must be specified using the observed data, in order to estimate the operating model. The approximating model is denoted as $g_{\theta}$, where the subscript $\theta$ indicates the $U$-dimensional parameter vector, which in turn specifies the probability density function.

Considering a system model composed of $N$ observations $\left\{\mathbf{x}_{1}, \mathbf{x}_{2}, \ldots, \mathbf{x}_{N}\right\}$. The transmitted signal by a PU is convolved with a multi-path channel where Gaussian noise is added. The received signal at a sensor node (i.e., one observation), denoted by the complex vector $\mathbf{x} \in$ $\left\{\mathbf{x}_{1}, \mathbf{x}_{2}, \ldots, \mathbf{x}_{N}\right\}$, can be modeled as

$$
\mathbf{x}=\mathbf{A s}+\mathbf{n}
$$

where $\mathbf{A}$ is the channel matrix whose columns are determined by the unknown parameters associated with each signal. $\mathbf{s}$ is the PU transmitted signal and $\mathbf{n}$ is the corresponding complex, stationary, and Gaussian noise with zero mean. Let $p$ be the length of one observation $\mathbf{x}$ and $q$ the length of the transmitted signal $\mathbf{s}$ and the 
additive noise $\mathbf{n}$. Our goal within this part is to determine the value of $q$ from $N$ observations (i.e., the dimension of the PU received signal). The number of signals $q$ is determined from the estimated covariance matrix $\hat{\mathbf{R}}$ defined by:

$$
\hat{\mathbf{R}}=\frac{1}{N} \sum_{n=1}^{N} \mathbf{x}_{n} \mathbf{x}_{n}^{N}
$$

The first step of the proposed sensing algorithm is the calculation of the covariance matrix $\hat{\mathbf{R}}$ of received $N$ signals. Then, we obtain the eigenvalues of this matrix $\left(\hat{\lambda}_{1}, \hat{\lambda}_{2}, \ldots, \hat{\lambda}_{p}\right)$ through eigenvalue decomposition technique, and we compute finally AIC and MDL values to estimate the dimension of the PU signal; The minimum of these values gives the number of significant eigenvalues. The AIC criterion is a widely used tool for model selection. Assuming a candidate model $\boldsymbol{g}_{\hat{\theta}}$, the idea is to decide if the observed model fits this candidate model. This criterion is defined by:

$$
\mathrm{AIC}=-2 \sum_{n=1}^{N} \log g_{\hat{\theta}}\left(\mathbf{x}_{n}\right)+2 U
$$

Inspired by Akaike work, Schwartz [14] and Rissanen [19] have an approach quite different. Schwartz [14], approached the problem by Bayesian arguments. However Rissanen based his study on information theoretic arguments [19]. It turns out that in the large-sample limit, both Schwartz's and Rissanen's approaches yield the same criterion, given by [20]:

$$
\mathrm{MDL}=-\sum_{n=1}^{N} \log g_{\hat{\theta}}\left(\mathbf{x}_{n}\right)+2 U \log N
$$

Using the estimated eigenvalues of the covariance matrix $\hat{\mathbf{R}}$, the resulting cost functions AIC and MDL have the following forms:

$$
\begin{aligned}
& \operatorname{AIC}(k)=-2 \log \left(\frac{\prod_{i=k+1}^{p} \hat{\lambda}_{i}^{p-k}}{\frac{1}{p-k} \sum_{i=k+1}^{p} \hat{\lambda}_{i}}\right)^{(p-k) N}+2 k(2 p-k)(2.6) \\
& \operatorname{MDL}(k)=-\log \left(\frac{\prod_{i=k+1}^{p} \hat{\lambda}_{i}^{p-k}}{\frac{1}{p-k} \sum_{i=k+1}^{p} \hat{\lambda}_{i}}\right)^{(p-k) N}+\frac{k}{2}(2 p-k) \log N \quad(2.7)
\end{aligned}
$$

\section{Spectrum sensing algorithms}

The goal of spectrum sensing is to decide between the following two hypothesizes $[2,3]$ :

$$
\mathbf{x}=\left\{\begin{array}{lr}
\mathbf{n} & \mathrm{H}_{0} \\
\mathbf{A s}+\mathbf{n} & \mathrm{H}_{1}
\end{array}\right.
$$

We decide that a spectrum band is unoccupied if there is only noise, as defined in $\mathrm{H}_{0}$. On the other hand, once there exists a PU signal besides noise in a specific band, as defined in $\mathrm{H}_{1}$, we say that the band is occupied. Thus the probability of false alarm can be expressed as

$$
P_{\mathrm{FA}}=\operatorname{Pr}\left(\mathrm{H}_{1} \mid \mathrm{H}_{0}\right)=\operatorname{Pr}\left(\mathbf{x} \text { is present }-\mathrm{H}_{0}\right)
$$

The decision threshold is determined by using the required probability of false alarm $P_{\mathrm{FA}}$ given by (3.2). The threshold $\gamma$ for a given false alarm probability is determined by solving the equation

$$
P_{\mathrm{FA}}=\operatorname{Pr}\left(\Upsilon(\mathbf{x})>\gamma \mid \mathrm{H}_{0}\right)
$$

where $\Upsilon(\mathbf{x})$ denotes the test statistic for the given detector. In the following, we will describe two existing spectrum sensing algorithms. We select an example of feature spectrum sensing algorithms, the CD detector, and an example of blind sensing algorithms, the ED detector. These algorithms will serve as references when evaluating the novel approaches resulting from the research.

\subsection{ED algorithm}

Conventional energy detectors can be simply implemented like spectrum analyzers. The energy detector measures the received energy during a finite time interval and compares it to a predetermined threshold. The test statistic of the energy detector is

$$
\Upsilon_{\mathrm{ED}}(\mathrm{x})=\sum_{i=1}^{p}\left|x_{i}\right|^{2}
$$

The performance of the energy detector in AWGN is well known and can be written in closed form. The probability of false alarm is given by

$$
P_{\mathrm{FA} . \mathrm{ED}}=1-Q\left(\frac{2 \gamma_{\mathrm{ED}}}{\sigma^{2}}, p\right)
$$

where $Q$ denotes the cumulative distribution function [21] of a $\chi^{2}$ distributed random variable with $2 p$ degrees of freedom. $\gamma_{\mathrm{ED}}$ is the detection threshold of the ED and $\sigma^{2}$ is the noise variance [9].

\subsection{CD algorithm}

$\mathrm{CD}$ has received a considerable amount of attention in the literature. Recent bibliography on cyclostationarity, including a large number of references on cyclostationarity based detection, is provided in [22]. The CD algorithm used in this article was presented in [23]. We will 
give in this subsection a brief description of this algorithm.

The cyclic autocorrelation function at some lag $l$ and some cyclic frequency $\alpha$ can be estimated from samples $\mathbf{x}$ by

$$
\hat{r}_{l}(\mathbf{x}, \alpha)=\frac{1}{p-l} \sum_{n=0}^{p-l-1} x_{n+l} x_{n}^{*} e^{-j \alpha n} l \geq 0
$$

The cyclic autocorrelations are non-zero for cyclostationarity based PU. This property is exploited to detect a PU by testing whether the expected value of the estimated cyclic autocorrelation is zero or not. Ghozzi et al. [23], introduce a detector based on multiple cyclic frequencies. Assuming that $\mathbf{s}$ is cyclic with cycle frequency $\alpha$

$$
\hat{\mathbf{r}}=\left[\operatorname{Re}\left\{\hat{r}_{l_{1}}(\alpha)\right\}, \ldots, \operatorname{Re}\left\{\hat{r}_{l_{K}}(\alpha)\right\}, \operatorname{Im}\left\{\hat{r}_{l_{1}}(\alpha)\right\}, \ldots, \operatorname{Im}\left\{\hat{r}_{l_{K}}(\alpha)\right\}\right]
$$

denotes a $1 \times 2 K$ vector containing the real and imaginary parts of the estimated cyclic autocorrelations for $K$ time delays at the cyclic frequency stacked in a single vector [23]. The test statistic is given by [23]

$$
\Upsilon_{\mathrm{CD}}(\mathbf{x})=\widehat{\mathbf{r}} \hat{\Sigma}^{-1} \widehat{\mathbf{r}}^{T}
$$

where $\hat{\Sigma}$ is an estimate of the covariance matrix $\Sigma \cong \operatorname{cov}\{\hat{\mathbf{r}}\}[23]$. The (asymptotic) probability of false alarm for this detector with threshold $\gamma_{\mathrm{CD}}$ is given by

$$
P_{\mathrm{FA}, \mathrm{CD}}=1-G\left(\frac{\gamma_{\mathrm{CD}}}{2}, K\right)
$$

where $G($.$) is the (lower) incomplete gamma function$ [23]. The main advantage of the cyclic autocorrelation function is that it differentiates the noise energy from the modulated signal energy.

\subsection{DED algorithm}

Zayen et al. [12], demonstrate that the number of DoF, possibly the number of significant eigenvalues, is determined as the value of $k \in\{0,1, \ldots, p-1\}$ which minimizes the value of AIC and/or the value of MDL. Figures 1a,b present the computed number of DoF obtained by AIC and MDL criterion following (2.6) and (2.7), respectively. We show in these two figures the behavior of the AIC and MDL curves as function of the eigenvalues index for an occupied and vacant UMTS band, respectively. The dimension of the covariance matrix is equal to 800 (i.e., $N=800$ observations) and the length of the received signals is 20480 samples. Based on (2.6) and (2.7), we determine the minimum of AIC and MDL and we obtain then the number of significant eigenvalues. From Figures 1a, we see clearly that the position of $\mathrm{AIC}_{\min }$ and $\mathrm{MDL}_{\text {min }}$ are located at a position of $k \neq 0$ for the occupied spectrum band, and,

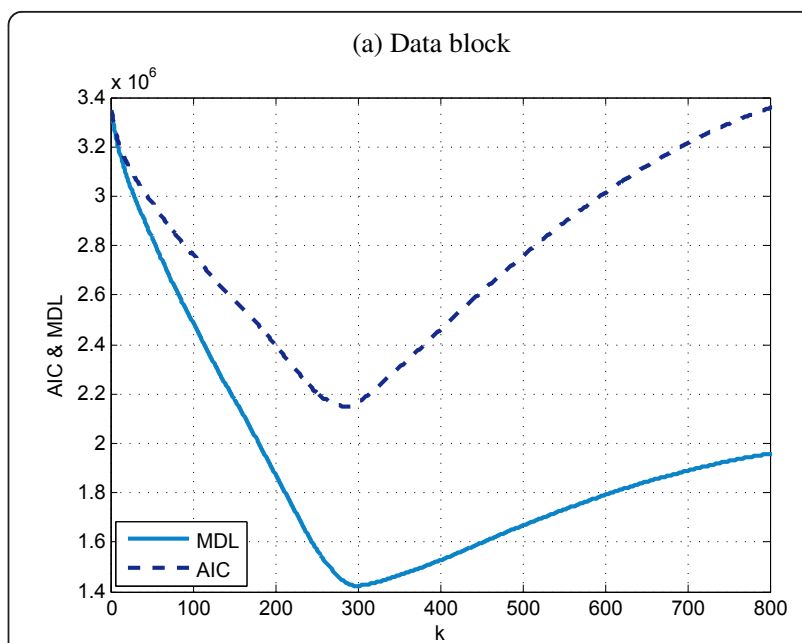

(b) Noise block

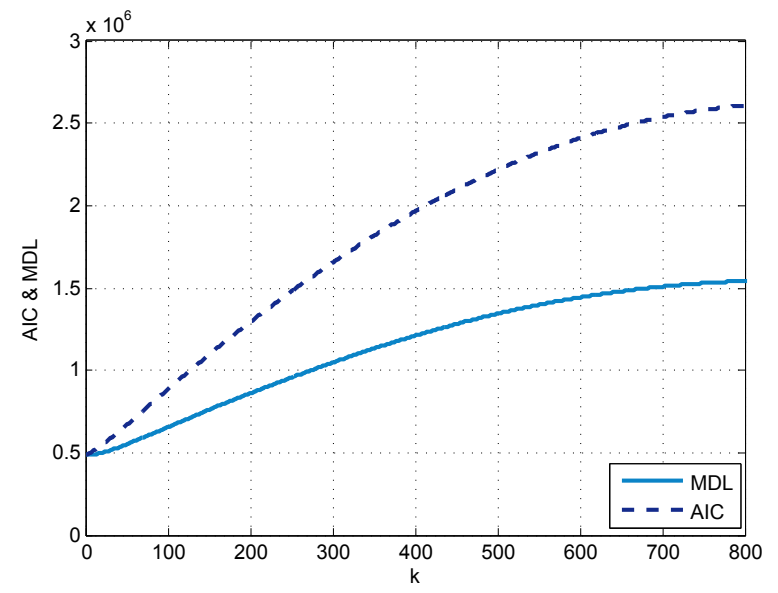

Figure 1 Akaike information criterion and minimum description length of captured noise block samples and data block samples using an UMTS signal. (a) Data block and (b) noise block.

at $k=0$ for the vacant spectrum band as given by Figures $1 \mathrm{~b}$. We tested also other communication signal types (GSM, WiFi, DVB-T OFDM with different channel models, etc.), and we found similar results.

Therefore, when the PU is absent, the received signal $\mathbf{x}$ is only the white noise samples, so the AIC curve, for example, monotonically increases. Therefore, $\operatorname{AIC}(0)<$ $\operatorname{AIC}(k), \forall k \in\{1, \ldots, p-1\}$, which can be rewired as AIC $(0)<\operatorname{AIC}(1)$. On the other hand, when the PU is present, the AIC curve monotonically decreases from AIC (0) to AICmin. Similarly, we can write that $\operatorname{AIC}(0)>$ $\mathrm{AIC}(1)$ if $\mathrm{PU}$ is present. Hence, the generalized blind DED using AIC criteria can be given by

$$
\Upsilon_{\mathrm{AIC}}(\mathrm{x})=\left\{\begin{array}{l}
\operatorname{AIC}(0)-\operatorname{AIC}(1)<\gamma_{\mathrm{AIC}} \mathrm{H}_{0} \\
\operatorname{AIC}(0)-\operatorname{AIC}(1)>\gamma_{\mathrm{AIC}} \mathrm{H}_{1}
\end{array}\right.
$$


The same properties can be founded using MDL criteria and the DED static test is given in this case by

$$
\Upsilon_{\mathrm{MDL}}(\mathbf{x})=\left\{\begin{array}{l}
\operatorname{MDL}(0)-\operatorname{MDL}(1)<\gamma_{\mathrm{MDL}} \mathrm{H}_{0} \\
\operatorname{MDL}(0)-\operatorname{MDL}(1)>\gamma_{\mathrm{MDL}} \mathrm{H}_{1}
\end{array}\right.
$$

We define here the two thresholds $\gamma_{\mathrm{AIC}}$ and $\gamma_{\mathrm{MDL}}$ in order to decide on the nature of the received signal. These thresholds depend only on $P_{\mathrm{FA}}$ and are calculated in the following section. In [17], does not involve any threshold value. A simple comparison between $\mathrm{AIC}(0)$ and AIC(1) makes the decision about the presence or absence of PU signal. The threshold value was neglected, i.e., at hypothesis $\mathrm{H}_{0}$ for example, $\Upsilon_{\text {AIC }}(\mathbf{x})=$ $\operatorname{AIC}(0)<\operatorname{AIC}(1)$.

\section{False alarm probability computation}

A theoretical probability of false alarm will be derived in this section using AIC and MDL criterion. The analytical results will be compared with simulation results to confirm the theoretical expression of thresholds and probabilities of false alarm.

\subsection{DED-AIC false alarm probability}

According to the sensing steps in Section 3, the false alarm of the DED using AIC criteria occurs when the estimated AIC values verify (3.10) given that the PU is absent or present. The test static $\Upsilon_{\mathrm{AIC}}(\mathbf{x})$ of the proposed detector is

$$
\Upsilon_{\mathrm{AIC}}(\mathbf{x})=\operatorname{AIC}(0)-\mathrm{AIC}(1)
$$

Therefore, the probability of false alarm can be expressed as

$$
P_{\mathrm{FA}, \mathrm{AIC}} \approx \operatorname{Pr}\left(\mathrm{AIC}(0)-\mathrm{AIC}(1)>\gamma_{\mathrm{AIC}} \mid \mathrm{H}_{0}\right)
$$

According to the AIC function defined in (2.6), we can obtain

$$
\begin{aligned}
& P_{\mathrm{EAAAC}}=\operatorname{Pr}\left(-2 \log \left(\frac{\prod_{i=1}^{p} \frac{1}{\hat{i}_{i}^{p}}}{\frac{1}{p} \sum_{i=1}^{p} \hat{\lambda}_{i}}\right)^{p^{\mathrm{N}}}+2 \log \left(\frac{\prod_{i=2}^{p} \frac{1}{\hat{\lambda}_{i}^{p-1}}}{\frac{1}{p-1} \sum_{i=2}^{p} \hat{\lambda}_{i}}\right)^{(p-1) N}-4 p+2>\gamma_{\mathrm{ACC}} \mid \mathrm{H}_{0}\right) \\
& =\operatorname{Pr}\left(\log \left(\frac{\left(\frac{1}{p} \sum_{i=1}^{p} \hat{\lambda}_{i}\right)^{p}}{\left(\frac{1}{p-1} \sum_{i=2}^{p} \hat{\lambda}_{i}\right)^{p-1} \hat{\lambda}_{1}}\right)>\frac{4 p-2+\gamma_{\text {AIC }}}{2 N} \mid \mathrm{H}_{0}\right)
\end{aligned}
$$

and at hypothesis $\mathrm{H}_{0}$ we have

$$
\frac{1}{p} \sum_{i=1}^{p} \hat{\lambda}_{i} \approx \frac{1}{p-1} \sum_{i=2}^{p} \hat{\lambda}_{i} \approx \sigma^{2}
$$

Infact, the sum of the eigenvalues of the estimated covariance matrix $\hat{\mathbf{R}}$, given by (4.16), is equivalent to $\frac{1}{\mathrm{pN}} \operatorname{Tr}\left(\sum_{n=1}^{N} \mathbf{x}_{n} \mathbf{x}_{n}^{H}\right)$. At hypothesis $\mathrm{H}_{0}$, the received vector involves only the noise samples, thats why, $\frac{1}{\mathrm{pN}} \operatorname{Tr}\left(\sum_{n=1}^{N} \mathbf{x}_{n} \mathbf{x}_{n}^{H}\right)$ is the unbiased estimation of the covariance of the white noise and it is equivalent to $\sigma^{2}$. Substituting now (4.16) into (4.15) yields:

$$
\begin{aligned}
P_{\mathrm{FA}, \mathrm{AIC}} & =\operatorname{Pr}\left(\frac{\sigma^{2 p}}{\sigma^{2 p-2} \hat{\lambda}_{1}}>\exp \left(\frac{4 p-2+\gamma_{\mathrm{AIC}}}{2 N}\right) \mid \mathrm{H}_{0}\right) \\
& =\operatorname{Pr}\left(\frac{\hat{\lambda}_{1}}{\sigma^{2}}<\exp \left(\frac{2-4 p-\gamma_{\mathrm{AIC}}}{2 N}\right) \mid \mathrm{H}_{0}\right)
\end{aligned}
$$

Let

$$
\mu=(\sqrt{N}+\sqrt{p})^{2}
$$

and

$v=(\sqrt{N}+\sqrt{p})\left(\frac{1}{\sqrt{N}}+\frac{1}{\sqrt{p}}\right)^{\frac{1}{3}}$. Then $\frac{N \frac{\hat{\lambda}_{1}}{\sigma^{2}}-\mu}{v}$ converges, with probability one, to the Tracy-Widom distribution of order two [18]. The false alarm probability can be rewritten as

$$
P_{\mathrm{FA}, \mathrm{AIC}}=\operatorname{Pr}\left(\frac{N \frac{\hat{\lambda}_{1}}{\sigma^{2}}-\mu}{v}<\frac{N \exp \left(\frac{2-4 p-\gamma_{\mathrm{AIC}}}{2 N}\right)-\mu}{v} \mid \mathrm{H}_{0}\right)
$$

Let $F_{2}$ denotes the CDF for the distribution of TracyWidom of order two given by [18]:

$$
F_{2}(t)=\exp \left(-\int_{t}^{\infty}(u-t) h^{2}(u) d u\right)
$$

where $h(u)$ is the solution of the nonlinear Painleve II differential equation [18]:

$$
h(u)=u h(u)+2 h^{3}(u)
$$

Therefore, the probability of false alarm of the DED algorithm using AIC criteria can be approximated as

$$
P_{\mathrm{FA}, \mathrm{AIC}}=F_{2}\left(\frac{N \exp \left(\frac{2-4 p-\gamma_{\mathrm{AIC}}}{2 N}\right)-\mu}{v}\right)
$$

or, equivalently

$$
\frac{N \exp \left(\frac{2-4 p-\gamma_{\mathrm{AIC}}}{2 N}\right)-\mu}{v}=F_{2}^{-1}\left(P_{\mathrm{FA}, \mathrm{AIC}}\right)
$$

we finally obtain the threshold

$$
\gamma_{\mathrm{AIC}}=2-4 p-2 N \ln \left(\frac{\nu F_{2}^{-1}\left(P_{\mathrm{FA}, \mathrm{AIC}}\right)+\mu}{N}\right)
$$

Generally, it is difficult to evaluate the function $F_{2}$. Fortunately, it can be computed using Matlab [18]. 


\subsection{DED-MDL false alarm probability}

Similar with the above derivation, when the MDL criterion is applied, we only need to modify the step in (4.15) as the one given in the following equation

$$
\begin{aligned}
& P_{\text {EAMMDL }}=\operatorname{Pr}\left(-\log \left(\frac{\prod_{i=1}^{p} \hat{\lambda}_{i}^{\frac{1}{p}}}{\frac{1}{p} \sum_{i=1}^{p} \hat{\lambda}_{i}}\right)^{p N}+\log \left(\frac{\prod_{i=1}^{p} \lambda_{i}^{\frac{1}{p-1}}}{\frac{1}{p-1} \sum_{i=2}^{p} \hat{\lambda}_{i}}\right)^{(p-1) N}-\left(p-\frac{1}{2}\right) \log N>\gamma_{\text {MDL }} \mid H_{0}\right) \\
& =\operatorname{Pr}\left(\log \left(\frac{\left(\frac{1}{p} \sum_{i=1}^{p} \hat{\lambda}_{i}\right)^{p}}{\left(\frac{1}{p-1} \sum_{i=2}^{p} \hat{\lambda}_{i}\right)^{p-1} \hat{\lambda}_{1}}\right)>\frac{\gamma_{\text {MDL }}+\left(p-\frac{1}{2}\right) \log N}{N}{ }_{H_{0}}\right)
\end{aligned}
$$

We consider the same supposition given by (4.16), where the received signal involves only the noise samples. Therefore, (4.24) can be written as

$$
P_{\mathrm{FA}, \mathrm{MDL}}=\operatorname{Pr}\left(\frac{\hat{\lambda}_{1}}{\sigma^{2}}<\exp \left(\frac{\gamma_{\mathrm{MDL}}+\left(p-\frac{1}{2}\right) \log N}{N}\right) \mid \mathrm{H}_{0}\right)
$$

Using the Tracy-Widom proposition, the false alarm probability of the DED algorithm using MDL criteria can be rewritten as

$$
P_{\mathrm{FA}, \mathrm{MDL}}=F_{2}\left(\frac{N \exp \left(\frac{\gamma_{\mathrm{MDL}}+\left(p-\frac{1}{2}\right) \log N}{N}\right)-\mu}{v}\right)
$$

where $\mu$ and $v$ are defined in the previous section, and the threshold of the DED-MDL algorithm is given by

$$
\gamma_{\mathrm{MDL}}=\left(p-\frac{1}{2}\right) \log N-N \ln \left(\frac{\nu F_{2}^{-1}\left(P_{\mathrm{FA}, \mathrm{MDL}}\right)+\mu}{N}\right)
$$

\subsection{Simulation and analytical results comparison}

When deriving the probabilities of false alarm using AIC and MDL criterion, was assumed the assumption given by (4.16) at hypothesis H0. This assumption is known not to be correct, but it was argued that it should be sufficient to obtain good theoretical results for the probability of false alarm. Note that, for the DED the threshold is not related to noise power and is computed based only on $N, p$, and $P_{F A}$, irrespective of signal and noise, for the two cases using AIC and MDL criterion. The comparison results for threshold and $P_{F A}$ using AIC and MDL criterion are given in Table 1 . This table shows that the simulated false alarm and thresholds performance matches the theoretical results with a high degree of accuracy.

\section{Performances evaluation}

Actual sensing results and performance studies will be provided in this section in the case of non-cooperative
Table 1 Simulation and analytical results comparison

\begin{tabular}{lcccc}
\hline & $\boldsymbol{P}_{\mathrm{FA},}$ & $\boldsymbol{p}=\mathbf{1 0 0}$ & $\boldsymbol{p}=\mathbf{1 5 0}$ & $\boldsymbol{p}=\mathbf{2 0 0}$ \\
& $\mathrm{AIC}$ & $\mathbf{0 . 0 5 3 1}$ & $\mathbf{0 . 0 5 1 8}$ & $\mathbf{0 . 0 5 0 4}$ \\
\hline $\begin{array}{l}\text { Simulation } \\
\text { results }\end{array}$ & $P_{\mathrm{FA},}$ & 0.0549 & 0.0533 & 0.0520 \\
& $\mathrm{MDL}$ & & & \\
& $\gamma_{\mathrm{AIC}}$ & $3.857 \mathrm{e} 04$ & $2.590 \mathrm{e} 04$ & $2.152 \mathrm{e} 04$ \\
& $\gamma_{\mathrm{MDL}}$ & $3.613 \mathrm{e} 04$ & $2.097 \mathrm{e} 04$ & $1.956 \mathrm{e} 04$ \\
& $P_{\mathrm{FA}, \mathrm{AIC}}$ & 0.0500 & 0.0500 & 0.0500 \\
\hline Analytical & $P_{\mathrm{FA},}$ & 0.0500 & 0.0500 & 0.0500 \\
results & $\mathrm{MDL}$ & & & \\
& $\gamma_{\mathrm{AIC}}$ & $3.762 \mathrm{e} 04$ & $2.527 \mathrm{e} 04$ & $1.984 \mathrm{e} 04$ \\
& $\gamma_{\mathrm{MDL}}$ & $3.484 \mathrm{e} 04$ & $1.825 \mathrm{e} 04$ & $1.754 \mathrm{e} 04$ \\
\hline
\end{tabular}

and cooperative CR network. The evaluation framework for all simulations has been implemented in Matlab and all results are obtained as the average of a number of Monte Carlo simulations. For the Monte Carlo simulation, each signal block consists of one symbol which contains 2048 samples. 500 iterations are performed in the simulation. The primary system used is a DVB-T system. Its communications are considered as PU communications. DVB-T abbreviates digital television broadcast-terrestrial, and as the name implies it is a standard for wireless digital transmission of TV signals. The standard is administered by the European telecommunications standards institute (ETSI). The official ETSI web page can be found at [24]. The choice of the DVB-T PU system is justified by the fact that most of the PU systems utilize the OFDM modulation format. The channel models implemented are AWGN, Rician and Rayleigh channels. The latter two correspond to the two different types of propagation that have to be handled in practice, namely line-of-sight (LOS) and non-line-of-sight (NLOS). Slow fading is simulated by adding log-normal shadowing. The simulation scenarios are generated by using different combinations of parameters given in Table 2 .

Three different scenarios with different properties have been chosen to evaluate the spectral detection performance, subject to provide different attributes so that the performance can be assessed under different

Table 2 The transmitted DVB-T primary user signal parameters

\begin{tabular}{ll}
\hline Bandwidth & $8 \mathrm{MHz}$ \\
Mode & $2 \mathrm{~K}$ \\
Guard interval & $1 / 4$ \\
Channel models & Rayleigh/Rician $(\mathrm{K}=1)$ \\
Maximum Doppler shift & $100 \mathrm{~Hz}$ \\
Frequency-flat & Single path \\
Sensing time & $1.25 \mathrm{~ms}$ \\
Location variability & $10 \mathrm{~dB}$
\end{tabular}


conditions, aiming to provide fair conditions before making conclusions. OFDM is the modulation of choice for the three simulation scenarios to be used as evaluation tools in this report. In OFDM, a wideband channel is divided into a set of narrowband orthogonal subchannels. OFDM modulation is implemented through digital signal processing via to the FFT algorithm [25]. In Scenario 1, we use a DVB-T OFDM signal in an AWGN channel. It is assumed that the detection performance in AWGN will provide a good impression of the performance, but it is necessary to extend the simulations to include signal distortion due to multipath and shadow fading. Scenario 2 utilizes the same DVB-T OFDM signal as Scenario 1, but to make the simulations more realistic, the signal is subjected to Rayleigh multipath fading and shadowing following a log normal distribution in addition to the AWGN. The maximum Doppler shift of the channel is $100 \mathrm{~Hz}$ and the standard deviation for the log normal shadowing is $10 \mathrm{~dB}$. Since the fading causes the channel to be time variant, it is necessary to apply longer averaging than in Scenario 1 to obtain good simulation results. Thus the number of iterations in the Monte Carlo simulation is increased from 500 to 1000 . The third simulation scenario utilizes also a DVB-T OFDM signal in Rician multipath fading with shadowing. The K-factor for the Rician fading is 10 , which represents a very strong line of sight component. The maximum Doppler shift of the channel and the standard deviation for the log normal shadowing are the same as in the second scenario.

\subsection{Non-cooperative sensing evaluation}

In this section, we will assess the performance of the proposed detector in terms of PU signal detection using the binary hypothesis test expressed in (3.10) and (3.11) for the DED-AIC and the DED-MDL detectors, respectively. The results from these simulations can be seen in the batch Figure 2. The best performance is obtained from the CD detector. Subsequent is the DED using AIC criteria which has a performance in the range from approximately $0.5 \mathrm{~dB}$ to approximately $2.5 \mathrm{~dB}$ below the $\mathrm{CD}$ detector. The worst performance is displayed by the DED-MDL detector and ED. DED-MDL performs approximately $3 \mathrm{~dB}$ above DED-AIC, while ED differs from the DED-AIC curves with as much as approximately $8 \mathrm{~dB}$. In total, DED-MDL and ED can be seen to perform an average about $6 \mathrm{~dB}$ worse than the best performance, which is obtained by the CD detector. From Figure 2, we remark also that relative detection results for Scenarios 2 and 3 are to a large extent aligned with the results for Scenario 1. This is expected as the

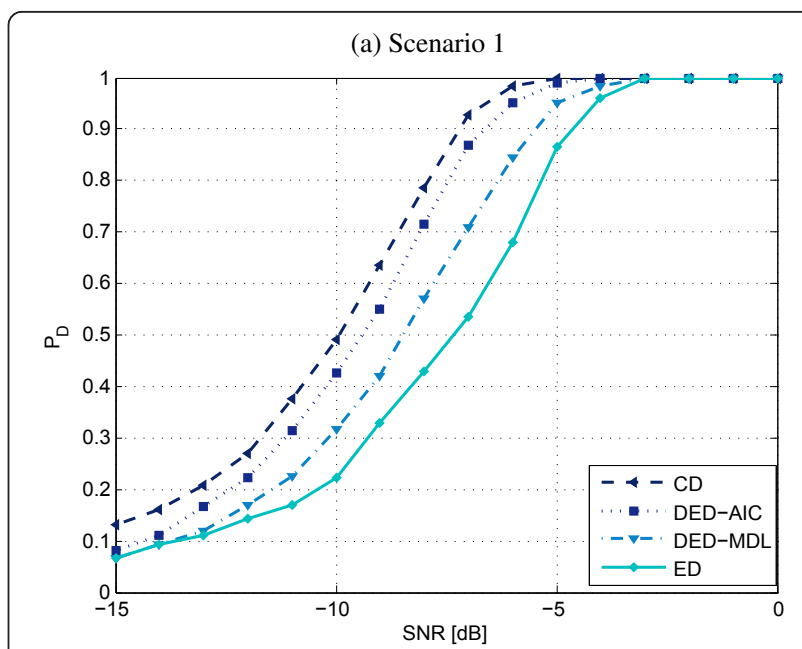

(b) Scenario 2

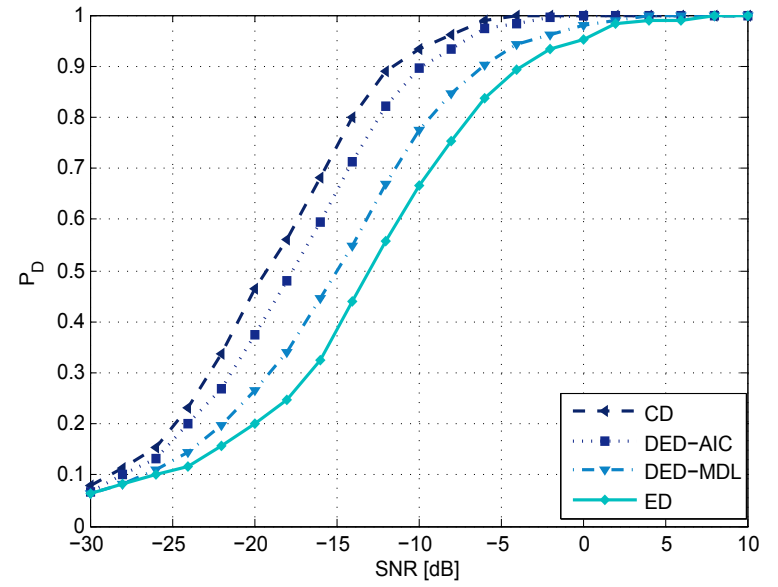

(c) Scenario 3

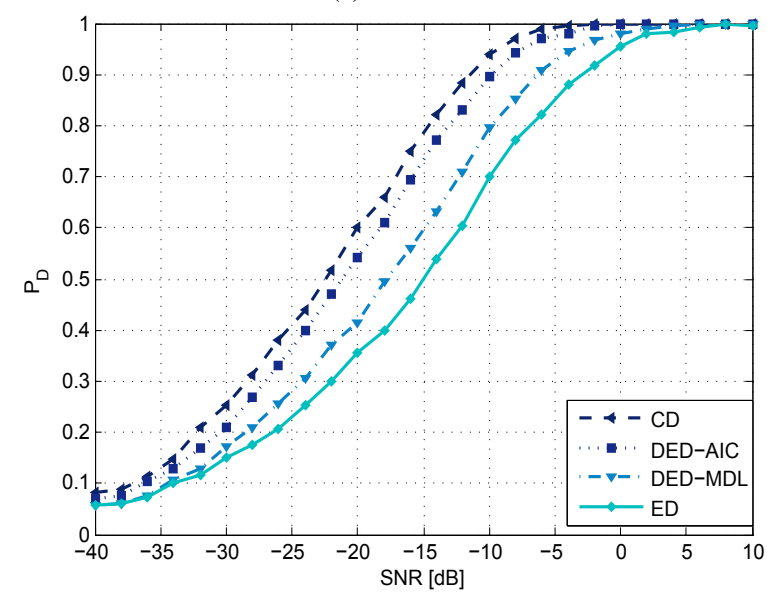

Figure 2 Performance evaluation of the DED detector in terms of PU signal detection using an DVB-T OFDM PU system: Probability of detection versus SNR curves with $P_{\mathrm{FA}}=0.05$, sensing time $=1.12 \mathrm{~ms}$ and $p=2048$. (a) Scenario 1 , (b) Scenario 2, and (c) Scenario 3. 
underlying used signals are the same. The main difference is in absolute performance which is caused by the addition of multipath and shadow fading.

It is obvious from Figure 2b,c how the absolute detection performance deteriorates when the signal is subjected to channel fading. The $P_{D}$ slope for all the detectors starts dropping at higher SNR values than for the AWGN case. While the $P_{D}$ curves started dropping off in the range from approximately $-3 \mathrm{~dB}$ to about -5 $\mathrm{dB}$ for the four detectors in the AWGN channel of scenario 1 , all curves start dropping off before $8 \mathrm{~dB}$ under the fading applied in Scenarios 2 and 3.

In general, some detectors like $\mathrm{CD}$ assume that some information about PU signal are explicitly known and their performance is optimal or close to optimal when this assumption is valid. The performance of such detectors deteriorates rapidly even for small departures from the underlying assumptions. Nonparametric or robust detectors on the other hand make no such assumptions and maintain their reliable performance in all conditions. There is a trade-off between robustness and optimality and robust techniques exchange the optimality to reliability. We developed in this work a realistic detector since it does not require any a priori knowledge about PU signal.

\subsection{Cooperative sensing evaluation}

The challenges of cooperative sensing include the development of efficient information sharing algorithms and increased complexity. Cooperative sensing decreases also the probability of false alarms considerably. In addition, cooperation can solve the hidden PU problem and can decrease sensing time. It can also mitigate the multipath fading and shadowing effects, which improve the detection probability. However, the cooperation causes adverse effects on resource-constrained networks due to the additional operations and overhead traffic.

In this article the cooperative spectrum sensing is performed as follows:

Step 1. Every SU performs local spectrum measurements independently and then makes a binary decision.

Step 2. All the SUs forward their binary decisions to a FC.

Step 3. The FC combines those binary decisions and makes a final decision to infer the absence or presence of the PU in the observed band.

In the above mentioned cooperative spectrum sensing algorithms, each cooperative partner makes a binary decision based on its local observation and then

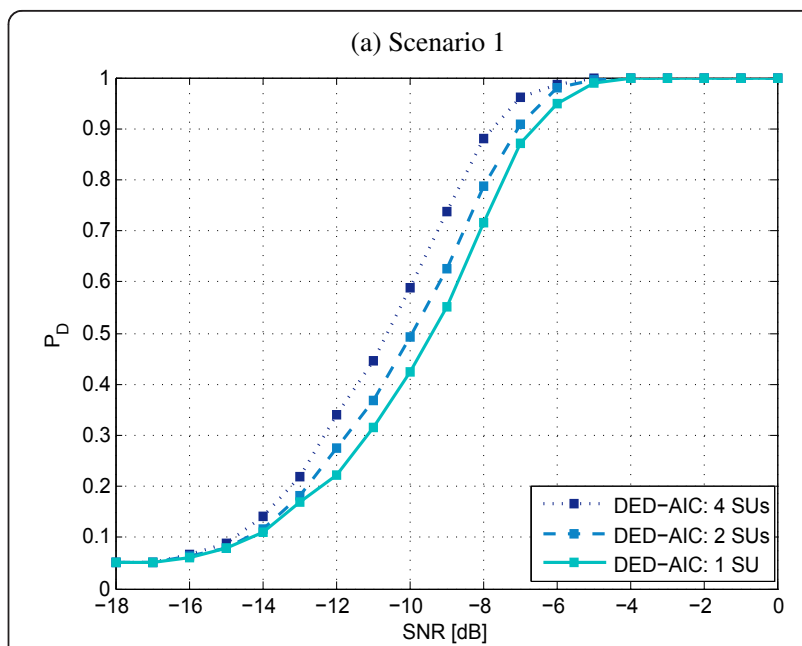

(b) Scenario 2

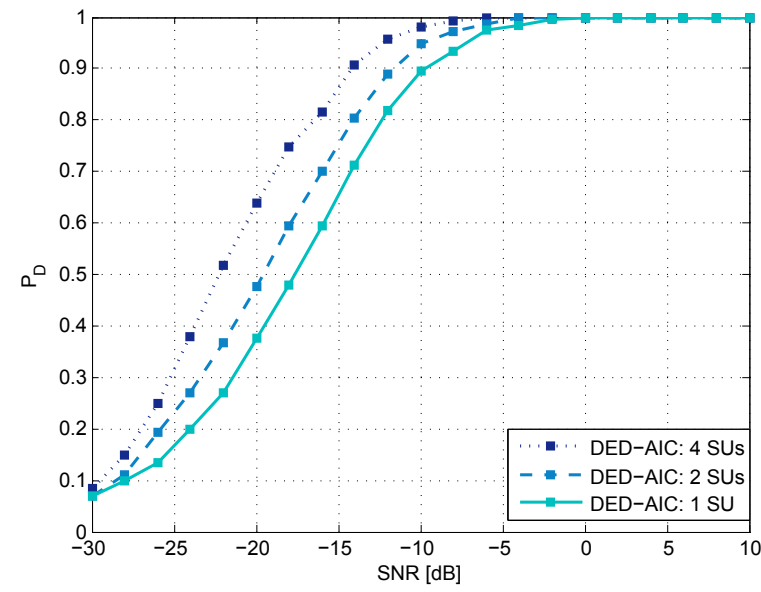

(c) Scenario 3

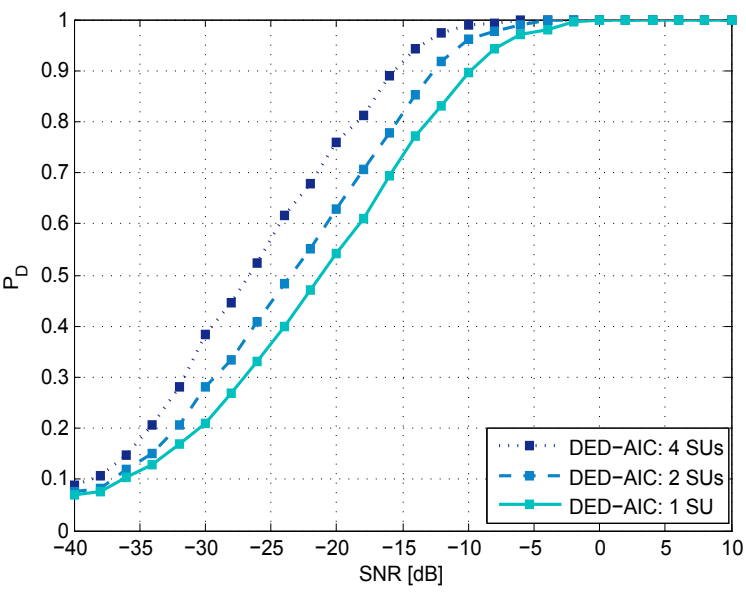

Figure 3 Performance evaluation of the DED detector in terms of PU signal detection in cooperative way using an DVB-T OFDM primary user system: Probability of detection versus SNR curves with $P_{\mathrm{FA}}=0.05$, sensing time $=1.12 \mathrm{~ms}$ and $p=$ 2048. (a) Scenario 1, (b) Scenario 2, and (c) Scenario 3. 
forwards one bit of the decision to the FC. At the FC, all one-bit decisions are fused together according to an "OR" logic. This cooperative sensing algorithm is referred to as decision fusion.

Results from the simulations can be seen in the batch Figure 3. These figures show the impact of cooperative SUs number $M$ in the detection performance. We plot the SNR for different numbers of cooperative users $M$, over the three scenarios. The false alarm probability is set to 0.05 . From the presented curves we can see that the cooperative sensing $(M>1)$ does increase the detection probability to its single user counterpart $(M=1)$, and the performance enhancement depends largely on the number of cooperative users. When $M$ increases, the performance is getting better.

\subsection{Complexity study}

This section provides a brief discussion on computational complexity of the DED algorithm. In order to give an idea of the complexity of the DED algorithm, we provide in Figure 4 simulation results assessing the performance in terms of execution time of this algorithm in comparison with $\mathrm{CD}$ and ED algorithms. Execution time has been measured by using the Matlab stopwatch function tic/toc. Simulations were performed on a laptop computer with a $1.6 \mathrm{GHz} \mathrm{CPU}$. From these results, we find that the $\mathrm{CD}$ is the most complex among all, with over two time complexity compared to DED. The ED is the least complex among all compared spectrum sensing algorithms.

Complexity terminology will be the asymptotic $\mathcal{O}$ -notation, which is standard when analyzing algorithms [26]. The complexity of the DED algorithm is computed

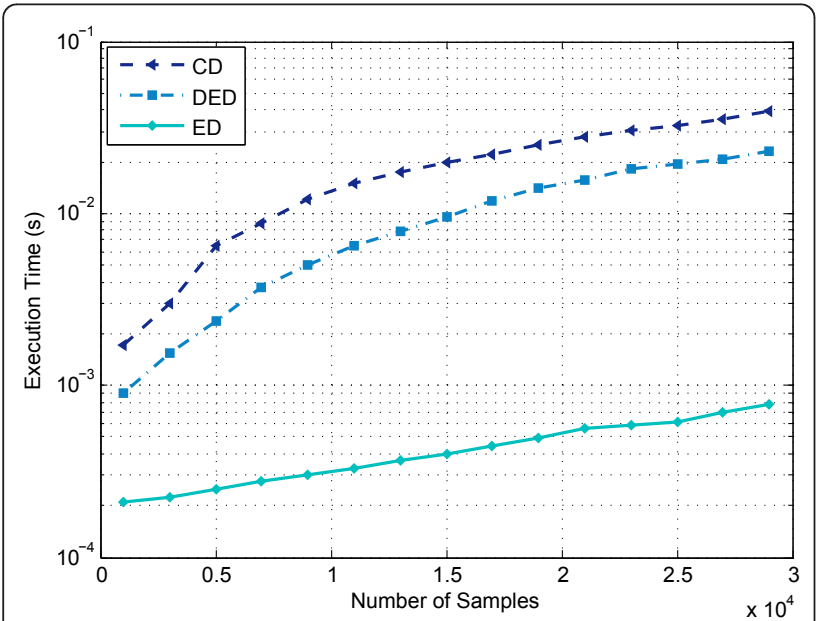

Figure 4 Simulation results assessing the performance in terms of execution time for the DED detector: Execution time versus the number of samples of the received DVB-T OFDM primary user signal. according to the different steps of the algorithm, namely computation of the covariance matrix and its corresponding eigenvalues and the derivation of AIC and MDL criterion for the DED-AIC and DED-MDL algorithms, respectively. Note that the complexity of AIC and MDL equations are equivalent because of the same number of multiplication/addition in the two equations. From the algorithm given in Section 3, we remark that the major complexity of this method comes from the computation of the covariance matrix and the eigenvalue decomposition. The covariance matrix is block Toeplitz matrix and hermitian, then $N p$ multiplications are sufficient. For the computation of eigenvalues, $\mathcal{O}\left(p^{3}\right)$ multiplications are needed. MDL and AIC values are computed according to (2.6) and (2.7) with $N p^{2}$ multiplications. The total complexity of the DED algorithm is therefore

$$
N p^{2}+N p+\mathcal{O}\left(p^{3}\right)
$$

This section provided a discussion on the computational complexity of the DED algorithm. We summarize the number of multiplications required for DED, CD, and ED algorithms in Table 3. From this table, it was argued that the DED algorithm asymptotically should have a better running time than the $\mathrm{CD}$ algorithm. This argument was further strengthened by simulation results. The simulations also showed that the DED algorithm have running times of approximately one to two order of magnitudes greater than the ED algorithm.

\section{Conclusion}

In this article, we derived the exact threshold expressions of the dimension estimation based spectrum sensing using AIC and MDL criterion. This is based on the distribution of Tracy-Widom of order two. Simulations using three different scenarios with different properties DVB-T PU systems were presented in order to verify the derived threshold values based on the probability of detection performance. It has been shown that analytical and empirical results are coincide with each other.

This paper provided also a number of simulations aimed at assessing the performance of the proposed detectors in comparison with two reference detectors, ED and CD detectors. From the presented results, we show that the ED has lost its detecting ability when decreasing the SNR. For sufficiently low SNR, robust

Table 3 Complexity comparison of the different sensing techniques

\begin{tabular}{lc}
\hline Sensing method & Complexity \\
\hline CD & $p^{2}+\mathcal{O}(p \log (p))$ \\
ED & $p$ \\
DED & $N p^{2}+N p+\mathcal{O}\left(p^{3}\right)$ \\
\hline
\end{tabular}


detection becomes impossible. These results come from the fact that the theoretical analysis for the ED algorithm assumes the noise variance to be known, and the underlying noise to have a perfect stationary Gaussian distribution. This assumption does not hold. In reality, the noise variance will usually not be completely stationary. The assumption about the distribution of the noise is also known to be weak. On the other hand, we find that if knowledge of signal parameters is provided, the CD detector can still perform a high probability of detection. Since this type of detection requires a priori knowledge about the received signal, they are not blind. Therefore, a CD can perform better than other detectors in discriminating against noise due to its robustness to the uncertainty in noise power. However, it is computationally complex and requires a significantly long observation time. The proposed detectors however do not require any information about $\mathrm{PU}$ transmitted signal and can detect the presence or the absence of PU blindly. Blind detection of telecommunication signals in a radio band is very helpful in some CR environments; especially, when SU does not have enough information about the PU. Finally, to adopt a spectrum sensing algorithm for a given situation depends on the CR environments and the spectrum sensor characteristics.

\section{Acknowledgements}

The research leading to these results had received funding from the European Community's Seventh Framework Programme (FP7/2007-2013) under grant agreement SACRA $n^{\circ} 249060$, and the European projects ACROPOLIS and CROWN.

\section{Author details}

${ }^{1}$ Mobile Communications Department, EURECOM, Sophia Antipolis, France ${ }^{2}$ GREENTIC, Hassan II University, Casablanca, Morocco

\section{Competing interests}

The authors declare that they have no competing interests.

Received: 1 May 2011 Accepted: 24 February 2012

Published: 24 February 2012

\section{References}

1. J Mitola, GQ Maguire Jr, Cognitive radio: making software radios more personal. IEEE Personal Commun. 6(4), 13-18 (1999). doi:10.1109/98.788210

2. J Mitola, Cognitive radio for flexible mobile multimedia communications, in Mobile Multimedia Communications (MoMUC), New York, pp. 3-10 (1999)

3. J Mitola, Cognitive Radio: An Integrated Agent Architecture for Software Defined Radio, (Doctor of Technology, Royal Inst Technol (KTH), Stockholm, Sweden, 2000)

4. AV Dantawate, GB Giannakis, Statistical tests for presence of cyclostationarity. IEEE Trans Signal Process. 42(9), 2355-2369 (1994). doi:10.1109/78.317857

5. S Roy, NM Neihart, DJ Allstot, A parallel, multi-resolution sensing technique for multiple antenna cognitive radios, in International Symposium on Circuits and Systems (ISCAS), New Orleans, LA, USA, pp. 2530-2533 (2007)

6. ABJ Kokkeler, Q Zhang, GJM Smit, An efficient multi-resolution spectrum sensing method for cognitive radio, in 3rd International Conference on Communications and Networking in China, Hangzhou, China, pp. 1226-1229 (2008)
7. Z Tian, GB Giannakis, A wavelet approach to wideband spectrum sensing for cognitive radios. in Conf on Cognitive Radio Oriented Wireless Networks and Communications (CROWNCOM) 1-5 (2006)

8. MA Lagunas-Hernandez, MA Rojas, P Stoica, New spectral estimation based on filterbank for spectrum sensing. in IEEE International Conference on Acoustics, Speech and Signal Processing 3509-3512 (2008)

9. H Urkowitz, Energy detection of unknown deterministic signals. Proc IEEE. 55(5), 523-531 (1967)

10. T Yucek, $H$ Arslan, A survey of spectrum sensing algorithms for cognitive radio applications. IEEE Commun Surv Tutorials. 11(1), 116-130 (2009)

11. M Haddad, AM Hayar, H Fetoui, M Debbah, Cognitive radio sensing information-theoretic criteria based, in CrownCom, Orlando, USA, pp. 241-244 (2007)

12. B Zayen, A Hayar, K Kansanen, Blind spectrum sensing for cognitive radio based on signal space dimension estimation, in IEEE ICC, Dresden, Germany, pp. 1-5 (2009)

13. H Akaike, Information theory and an extension of the maximum likelihood principle, in ISIT, Springer Verlag 73(1), pp. 267-281 (1973)

14. G Schwartz, Estimating the dimension of a model. Ann Stat. 6, 497-511 (1978)

15. Y Zeng, YC Liang, Eigenvalue-based spectrum sensing algorithms for cognitive radio. IEEE Trans Commun. 57(6), 1784-1793 (2009)

16. YC Liang, TJ Lim, R Zhang, Y Zeng, Glrt-based spectrum sensing for cognitive radio. in IEEE GLOBECOM 1-5 (2008)

17. $\mathrm{R}$ Wang, $M$ Tao, Blind spectrum sensing by information theoretic criteria for cognitive radios. IEEE Trans Veh Technol. 59(8), 3806-3817 (2010)

18. IM Johnstone, On the distribution of the largest eigenvalue in principal components analysis. Ann Stat. 29(2), 295-327 (2001). doi:10.1214/aos/ 1009210544

19. J Rissanen, Modeling by shortest data description. Automatica. 14, 465-471 (1978). doi:10.1016/0005-1098(78)90005-5

20. M Wax, T Kailath, Detection of signals by information theoretic criteria. IEEE Trans ASSP. 33(2), 387-392 (1985). doi:10.1109/TASSP.1985.1164557

21. M Abramowitz, IA Stegun, Handbook of Mathematical Functions with Formulas, Graphs, and Mathematical Tables, 10th edn. (U.S. National Bureau of Standards, Washington, DC, 1972)

22. A Napolitano, WA Gardner, L Paura, Cyclostationarity: Half a century of research. Signal Process. 86(4), 639-697 (2006). doi:10.1016/j. sigpro.2005.06.016

23. M Ghozzi, F Marx, M Dohler, J Palicot, Cyclostatilonarilty-based test for detection of vacant frequency bands. in 1st IEEE International Conference on Cognitive Radio Oriented Wireless Networks and Communications 1-5 (2006)

24. Official Web Page, European telecommunications standards institute. http:// www.etsi.org

25. A Goldsmith, Wireless Communications, (Cambridge Univ Press New York 2005)

26. RL Rivest, TH Cormen, CE Leiserson, C Stein, Introduction to Algorithms, MIT Press Cambridge MA, pp. 141-145 (2001)

doi:10.1186/1687-1499-2012-64

Cite this article as: Zayen and Hayar: Dimension estimation-based spectrum sensing for cognitive radio. EURASIP Journal on Wireless Communications and Networking 2012 2012:64.

\section{Submit your manuscript to a SpringerOpen ${ }^{\circ}$ journal and benefit from:}

- Convenient online submission

- Rigorous peer review

- Immediate publication on acceptance

- Open access: articles freely available online

- High visibility within the field

- Retaining the copyright to your article

Submit your next manuscript at $>$ springeropen.com 